\title{
Resistance to drugs and cell death in cancer stem cells $(\mathrm{CSC} s)$
}

\author{
Ahmad R Safa* \\ Department of Pharmacology and Toxicology, Indiana University School of Medicine, Indianapolis, USA
}

\begin{abstract}
Human cancers emerge from cancer stem cells (CSCs), which are resistant to cancer chemotherapeutic agents, radiation, and cell death. Moreover, autophagy provides the cytoprotective effect which contributes to drug resistance in these cells. Furthermore, much evidence shows that CSCs cause tumor initiation, progression, metastasis, and cancer recurrence. Various signaling pathways including the phosphatidylinositol 3-kinase (PI3K)/Akt/mammalian target of rapamycin ( $\mathrm{mTOR}$ ), maternal embryonic leucine zipper kinase (MELK), NOTCH1, and Wnt/ $\beta$-catenin as well as the CSC markers maintain CSC properties. Several mechanisms including overexpression of ABC multidrug resistance transporters, a deficiency in mitochondrial-mediated apoptosis, upregulation of c-FLIP, overexpression of anti- apoptotic Bcl-2 family members and inhibitors of apoptosis proteins (IAPs), and PI3K/AKT signaling contribute to enhancing resistance to chemotherapeutic drugs and cell death induction in CSCs in various cancers. Studying such pathways may help provide detailed understanding of CSC mechanisms of resistance to chemotherapeutic agents and apoptosis and may lead to the development of effective therapeutics to eradicate CSCs.
\end{abstract}

\begin{abstract}
Abbreviations: Apaf-1: Apoptotic Proteinase-Activating Factor-1; AIC: Apoptosis Inhibitory Complex; BH3: Bcl-2 Homology Domain-3; CSCs: Cancer Stem Cells; c-FLIP: cellular FLICE (FADD-like IL$1 \beta$-converting enzyme)-Inhibitory Protein; DISC: Death-Inducing Signaling Complex; DNA-PK: DNA-Dependent Protein Kinase; FADD: Fas-Associated Death Domain; ER: Endoplasmic Reticulum; DRs: Death Receptors; GBM: Glioblastoma Multiforme; mTOR: Mammalian Target of Rapamycin; MELK: Maternal Embryonic Leucine Zipper Kinase; Nrf2: Nuclear Factor Erythroid 2-Related Factor 2; P-gp: P-glycoprotein; PDA: Pancreatic Ductal Adenocarcinoma; PTP: Permeability Transition Pore; PERK: Protein Kinase R (PKR)-like Endoplasmic Reticulum Kinase; TNFRl: TNF Receptor 1; TNF- $\alpha$ : Tumor Necrosis Factor- $\alpha$; TRAIL: TNF-Related Apoptosis-Inducing Ligand; TRADD: TNFR1-Associated Death Domain Protein (TRADD); UPR: Unfolded Protein Response; Hh: hedgehog.
\end{abstract}

\section{Introduction}

Various cancer types contain a highly heterogeneous cell population containing distinct subpopulations of cells with genetic, epigenetic, and morphologic variations. The heterogeneity and progression of malignancies are explained by two models: (a) the CSC or cancer initiating cell (ClC) model [1,2], and (b) the stochastic model or clonal evolutionary model [3]. The CSC model or hierarchical model describes that tumors arise from a small percentage of CSCs as a result of normal stem cell (NSC) mutations and strongly suggests that CSCs cause an orderly system generating the entire tumor cell population [1,2] (Figure 1). In the stochastic model, normal cells acquire mutations during their survival before they develop into CSCs and initiate tumorigenesis [3]. Moreover, CSC-like cells can be generated by epigenetic plasticity due to druginduced dedifferentiation or conversion of non-CSCs to CSCs, which make cancer therapy more complex [4] (Figure 1). However, in the clonal evolutionary model, each cell within the tumor possesses the potential to produce malignant tumors expressing various degrees of chemoresistant subpopulations (Figure 1).
Tumor recurrence is the major cause of death in patients with incurable malignancies and is attributed to treatment-resistant CSCs within the primary tumor (Figure 2). CSCs are a rare cell population within a tumor, which express differing molecular markers in various types of cancers $[1,2,4]$. Delineating the molecular characterization of CSC populations will allow us to identify the targeted agents that cause their cell death, thus increasing the development of more effective treatment regimens for cancer. In this model, tumors contain a very small population of tumor initiating cells termed CSCs or stem-like cells, which are responsible for the initiation, progression, metastasis, drug resistance, and recurrence of cancer $[1,2,4]$. These therapy resistant, quiescent and pluripotent cells reside in CSCs niches, which provide specific microenvironments that protect CSCs against cell death, chemotherapy, and radiotherapy $[5,6]$. Therefore, there is a hierarchy of cells within tumors, which is initiated from a CSC population. Tumors exhibit stemness (self-renewal and multilineage differentiation) because CSCs have the capacity to recapitulate xenografts similar to the original tumor $[5,6]$. The self-renewal and differentiation capacities lead to the production of various cancer cell types in tumors and thereby create tumor heterogeneity [7] with various degrees of resistance to different therapeutics.

Drug resistance has been a major impediment for successfully treating tumors, not only with conventional chemotherapeutics, but with targeted therapies as well [8-11]. A major contributor to drug resistance is cellular heterogeneity within a tumor. Another main limitation in cancer therapy has proven to be a lack of refractoriness to

*Correspondence to: Ahmad R. Safa, Department of Pharmacology and Toxicology, 635 Barnhill, Dr. MS A416, Indiana University School of Medicine, Indianapolis, IN, USA, E-mail: asafa@iupui.edu

Key words: cancer stem cells (CSCs), apoptosis, drug resistance, death receptor pathways, anti-apoptotic proteins, Bcl-2 family, c-FLIP

Received: June 10, 2019; Accepted: June 21, 2019; Published: June 24, 2019 


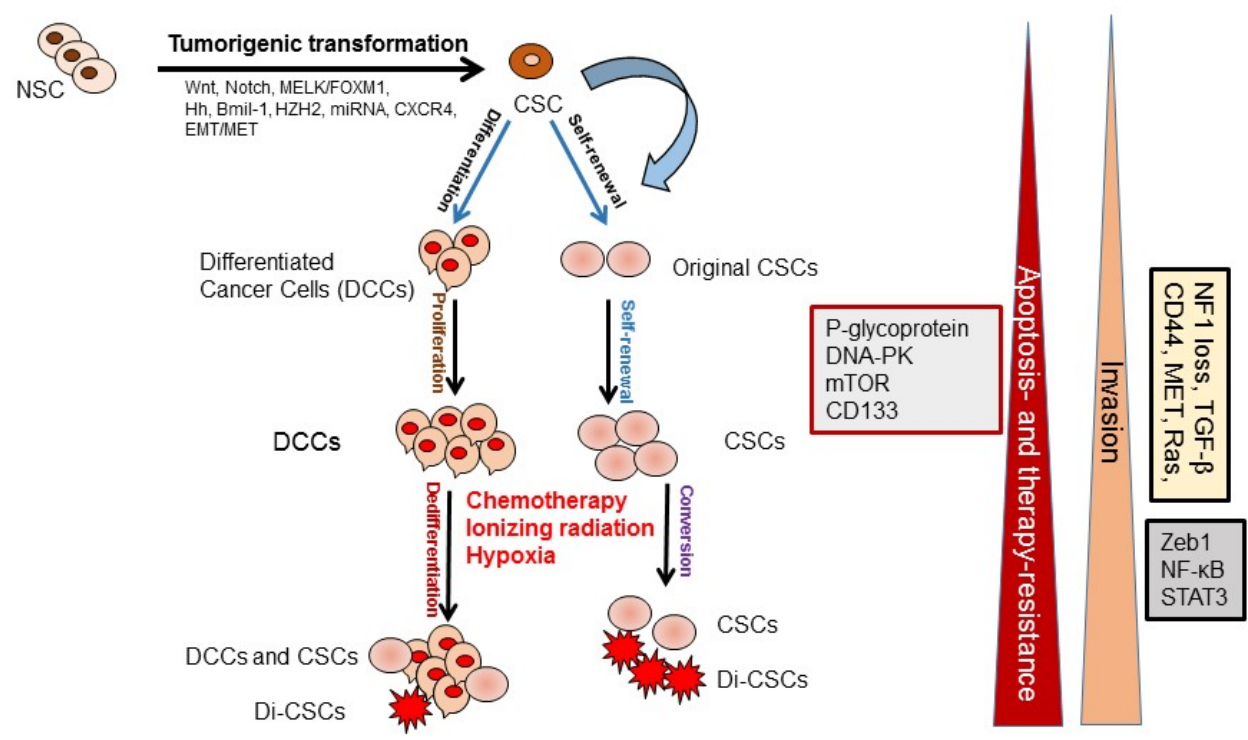

Figure 1. Cancer stem cells (CSCs) role in tumor development and progression. CSCs are generated from the normal stem cells (NSCs) through tumorigenic transformation of several potential pathways including Hh: hedgehog, epithelial-to-mesenchymal transition (EMT), and the reverse process mesenchymal-to-epithelial transition (MET). CSCs and drug-induced CSCs (Di- CSCs) are enriched following conventional chemotherapy treatment

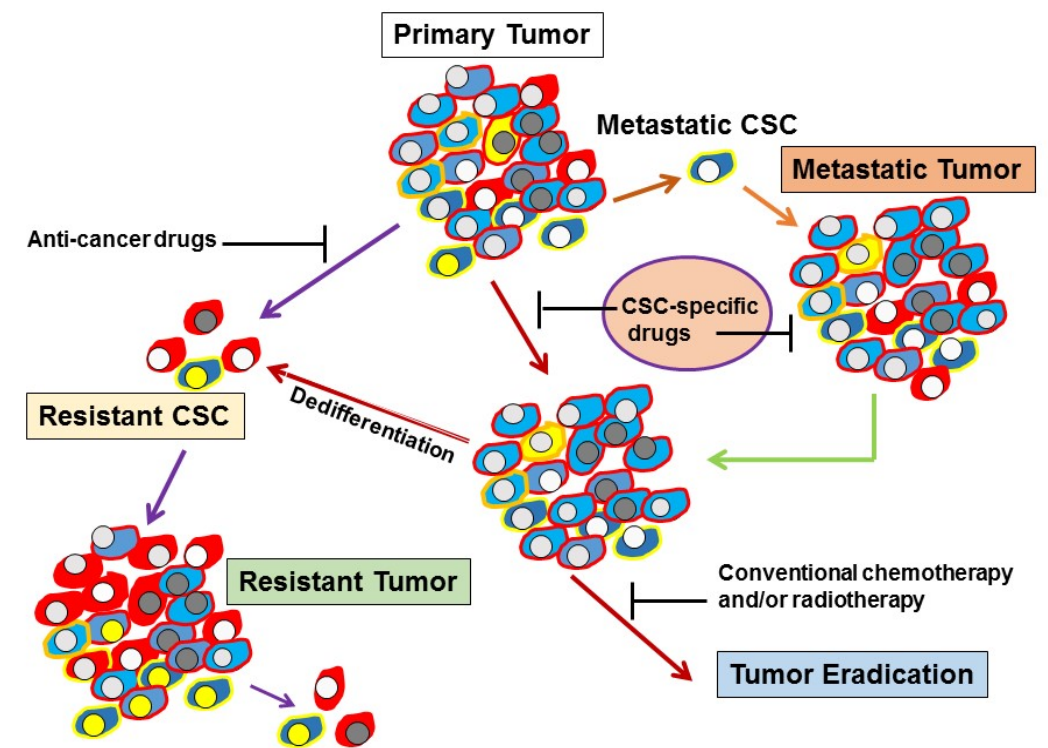

CancerStem Cells (CSCs)

Figure 2. Heterogeneity of CSCs in tumors. Development of drug resistant, metastatic tumors, and a potential strategy for eradicating tumors using CSC-specific drugs

apoptosis due to intrinsic resistance to cell death (e.g., pancreatic cancer, colon cancer, glioblastoma, and prostate cancer are typically refractory to cancer chemotherapy) or acquired resistance (e.g., after breast cancer chemotherapy). Much evidence has proven that within solid tumors, there are distinct populations of cancer cells contributing to the complexity of cancer treatment. Major contributors to intratumoral heterogeneity are cancer stem cells (CSCs), cellular genotype, genomic instability, epigenetic variation, cell plasticity, stochastic processes, and the microenvironment including distinct subpopulations of cancer associated fibroblasts (CAFs) and cancer-associated macrophages (CAMs) [12] causing various effects on cancer cells. Therefore, drug resistance in tumors is very complex and modulation or circumvention of drug resistance requires specific inhibitors for targeting relevant cellular targets.

Chemoresistance emerges due to several factors including environmental factors, pharmacodynamics, tumor heterogeneity in each patient as well as genetic and epigenetic alterations in malignant cells [13-17]. Many mechanisms participate in triggering resistance to chemotherapeutic drugs in cancer cells and characterizing these mechanisms will provide critical information for the design and development of more effective approaches to circumvent drug resistance in malignant cells and tumors. Deregulation of apoptotic and cell death signaling pathways and upregulation of survival mechanisms 
in cancer cells, particularly in CSCs, confer resistance to various drugs in a wide variety of cancers [18-22]. Additionally, epigenetic alterations can contribute to drug resistance and aggressive tumor behavior. Therefore, designing approaches to increase cell death in CSCs may lead to the development of useful therapeutics to eliminate these cells. Moreover, epigenetic plasticity is specifically critical in the interconversion of differentiated cells into CSCs and vice versa [4], which adds to the complexity of successfully treating cancers. Therefore, the major requirement for effective and successful cancer therapeutics is to eliminate CSCs, differentiated cancer cells, and the heterogeneous subpopulations of cancer cells in the entire tumor.

\section{Drug resistance in CSCs}

Strong evidence shows that CSCs are highly resistant to conventional chemotherapies [11,23-32]. Various drug resistance mechanisms have been reported in CSCs including increased antiapoptotic proteins such as Bcl-2 Bcl-X, and c-FLIP [11,26], high expression of ATP-binding cassette $(\mathrm{ABC})$ transporter proteins and detoxifying enzymes [26-28], cell cycle quiescence [29,30], increased DNA repair ability $[26,27]$, elevated aldehyde dehydrogenase (ALDH) activity [31], activation of key prosurvival signaling molecules such as NOTCH, Wnt/ $\beta$-catenin, and NF- $\kappa \mathrm{B}$ [32-34], increased activities of the phosphatidylinositol 3-kinase (PI3K)/Akt/mammalian target of rapamycin (mTOR), and maternal embryonic leucine zipper kinase (MELK) [11,35].

Recent findings show that CSCs are quiescent in the resting stage of the cell cycle and resistant to chemotherapy since most of these drugs target proliferating cells [36,37]. Upregulation of DNA repair proteins in CSCs correlates with rapid DNA repair, which also participates in drug and radiation resistance. [27,38-40]. Moreover, the cancer microenvironment (niche) protects CSCs from cancer therapy $[27,41]$ and CSCs contribute to the niche in a feedback loop and mutual manner $[32,41]$. The extracellular matrix (ECM) facilitates and maintains cancer stem cells and drug resistance [42]. In addition to delineating molecular and biochemical mechanisms of drug resistance, understanding and defining the crosstalk between CSCs and their niche is critical for overcoming resistance to anticancer drugs and cell death.

In this review article, I discuss the contribution of particular drug resistance mechanisms and signaling pathways that control CSC maintenance. Understanding these mechanisms is important for overcoming drug resistance in these cells [13-15,23,34]. The major mechanisms of chemotherapeutic and apoptotic resistance in CSCs are summarized in Figures 3 and 4.

\section{Cancer stem cells signaling pathways}

It is well documented that various cancers are initiated from CSCs and these cells are responsible for patient resistance to therapies [11,4346]. Furthermore, due to the heterogeneity, high diversity, and plasticity of CSCs, developing useful therapeutics to target them has been difficult. Evidence also suggests the possibility of non-CSC reprogramming and dedifferentiation to CSCs (Figure 1), resulting in a further increase in the complexity and diversity of resistance mechanisms in tumors. Therefore, because of this complexity, potent and effective anticancer treatment must eradicate both CSCs and the entire bulk of the heterogeneous tumor population, as well as avoid triggering tumor cell dedifferentiation of non-CSCs to CSCs or cancer stemlike cells. Various signaling pathways including the Notch, Hedgehog, Wnt/ $\beta$-catenin, PI3K/Akt/ mTOR (mTORC1 and mTORC2), maternal embryonic leucine zipper kinase (MELK), TGF- $\beta$, STAT, and HippoYAP/TAZ among others are activated and operational in CSCs [11,4649]. These pathways and the cancer stem cell markers including CD133, CD44, Oct4, Sox2, Nanog, and ALDH1A1 maintain CSC properties $[11,34,50,51]$.

Another important factor, epigenetic modification of CSCs, could result in phenotypic and functional heterogeneity among the cell populations within various solid tumors. Accumulating evidence indicates that the enhancer of zeste homolog $2(\mathrm{EZH} 2)$, which is the catalytic subunit of Polycomb repressive complex 2 (PRC2) and has

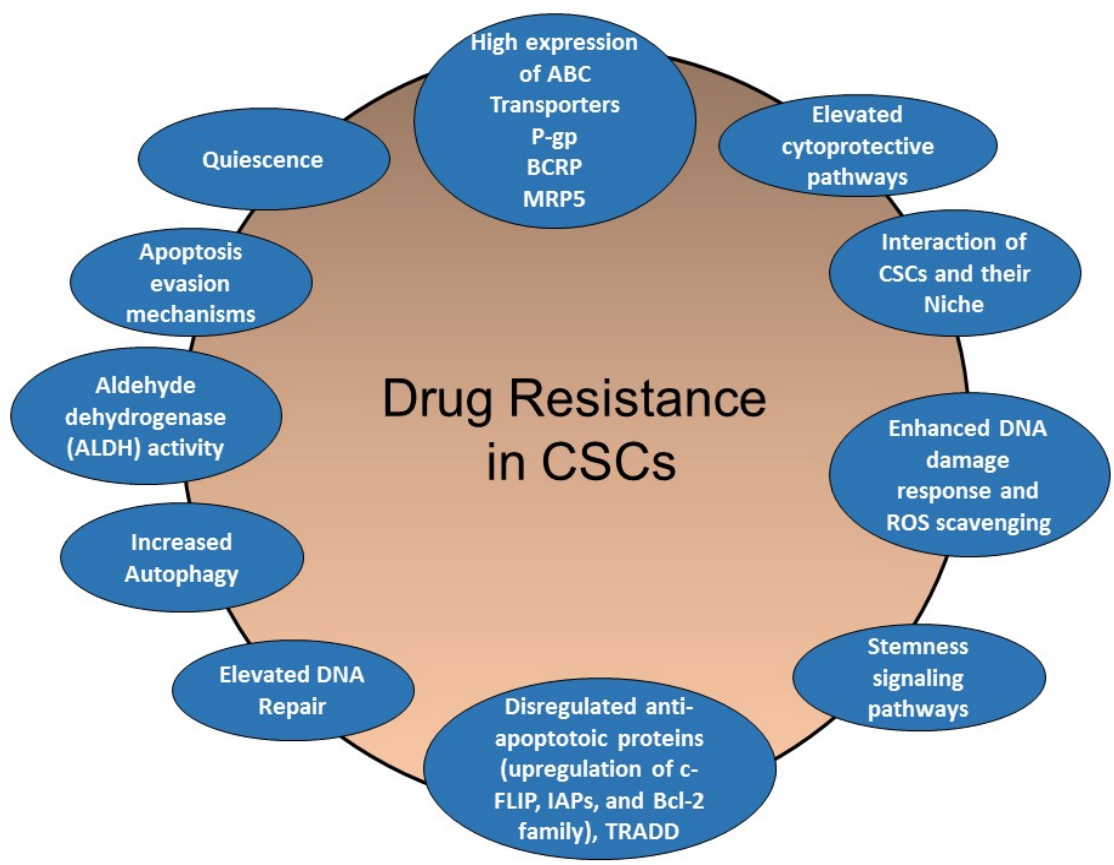

Figure 3. Schematic presentation of CSC-mediated therapy resistance to cancer. Activation of cell survival pathways, quiescence, increased drug efflux, impairment of the apoptotic pathway, increased DNA damage repair, increased detoxifying activity, and increased scavenging of free radicals are possible contributors to the therapy resistance of CSCs 


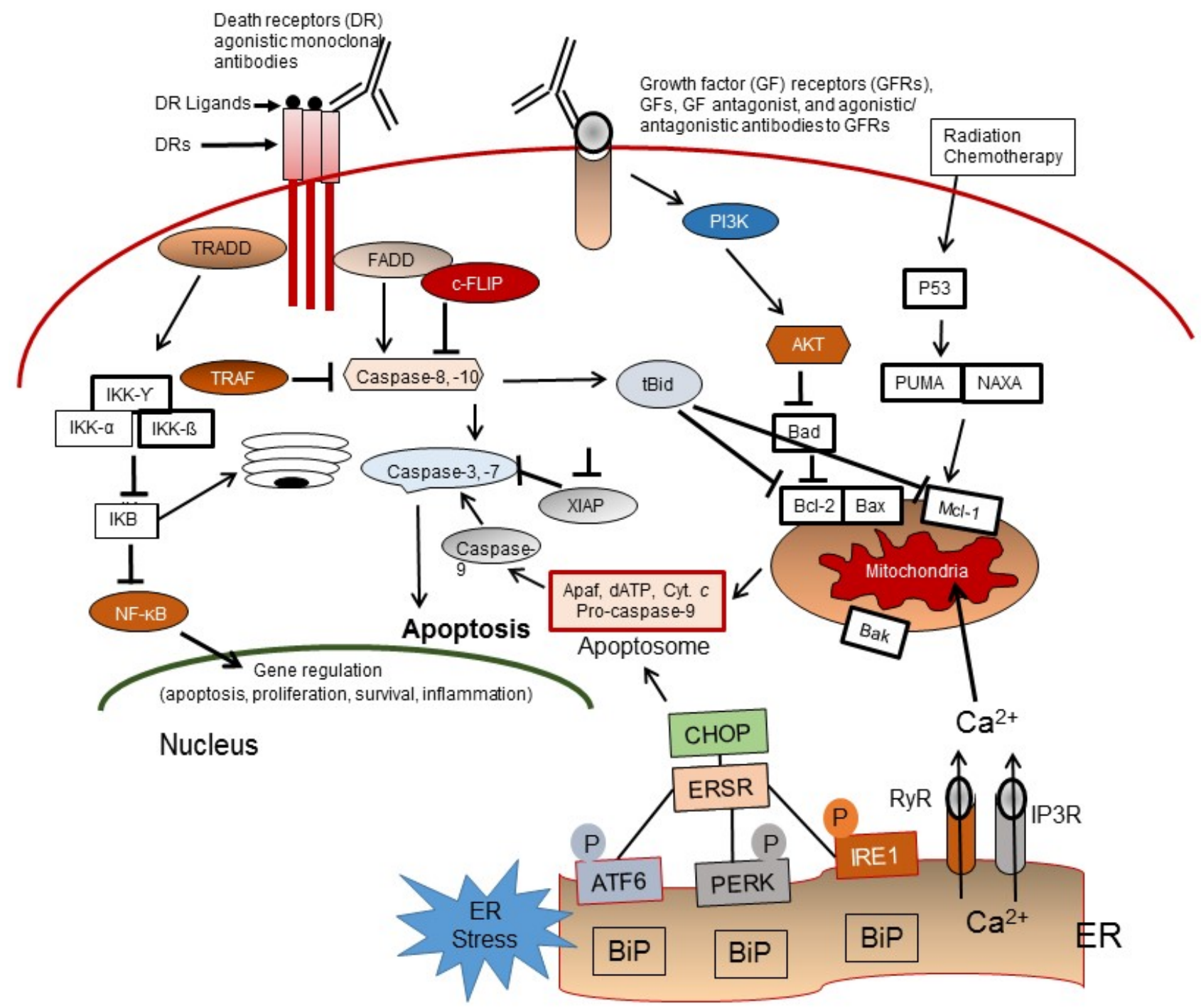

Figure 4. Apoptosis signaling pathways. Overview of the intrinsic (mitochondrial), extrinsic or death receptor (DR), and ER-stress (ERS)-mediated apoptosis pathways in response to the molecular action of anticancer agents as well as the TRADD/NF- $\kappa B$ survival pathway, the growth factor (GF) receptors, and PI3K/Akt pro-survival signaling axis in CSCs

histone methyltransferase activity, is upregulated in CSCs and has an important function in their expansion and maintenance [52]. Furthermore, histone deacetylases (HDACs) 1, 6, 7 and 8, known to deacetylate transcription factors and other cellular proteins, are overexpressed in CSCs $[53,54]$.

Significantly, it has been shown that that hypoxia-driven CSC enrichment results from a dedifferentiation process in breast cancer and that hypoxia-inducible factors (HIFs) are required for chemotherapy resistance in breast CSCs (BCSCs) [55], glioblastoma CSCs [56] and other solid tumors [57]. Interestingly, the dedifferentiated CSCs display multidrug resistance (MDR) via PERK-Nrf2 signaling pathway [58]. Lee et al. [56] have demonstrated that temozolomide (TMZ)-triggered HIF $1 \alpha / H I F 2 \alpha$ upregulation plays a critical role in converting non-stem glioma cells to acquire stem-like characteristics, and that knockdown of HIF1 $\alpha /$ HIF $2 \alpha$ suppressed the interconversion between non-stem glioma cells and GSCs post-therapy [56].

Another crucial signaling protein, MELK, a serine/threonine kinase, is upregulated in human cancers and CSCs [59] and plays significant roles in the survival and other known characteristics of
CSCs including drug resistance and tumor recurrence. Kim et al. [60] demonstrated that MELK phosphorylates the FOXM1 transcription factor and that EZH2 is targeted by the MELK/FOXM1 complex, which promotes CSC resistance to radiation, and that OTS167 (an inhibitor of MELK) effectively eliminates CSCs from small cell lung cancer (SCLC) [61].

The CSC characteristics that may help in the development of antiCSC therapies include specific cell surface markers and particular networks of transcription factor signaling, aberrant signaling pathways, epigenetic alterations, reprograming and plasticity, interaction with the microenvironment and CSC niche, and using particular metabolic pathways [25,43,60-64].

\section{Apoptosis pathways and resistance to apoptosis in CSCs}

Apoptosis resistance and overexpression of anti-apoptotic proteins are necessary for CSC survival and self-renewal capacity. To discuss the mechanisms of apoptosis and cancer-related chemotherapeutic drug resistance, apoptosis signaling pathways are first described. Cancer cells and CSCs avoid apoptosis, but apoptosis 
in these cells occurs through several signaling pathways in response to chemotherapeutic agents and various apoptotic stimuli. Much evidence shows that mutations in normal stem cells (NSCs) alter these cells into CSCs (Figure 1), enabling them to avoid apoptosis and leading to tumor formation.

Three major apoptosis pathways are the extrinsic or cell surface death receptors pathway, the intrinsic or mitochondrion-initiated pathway, and endoplasmic reticulum (ER) stress-mediated pathway control of apoptosis (Figure 3) [65-74]. The death receptor mediated or extrinsic apoptotic pathway is initiated by the binding of death receptors (DRs) with their ligands [i.e., interaction of Fas/Fas ligand, tumor necrosis factor- $\alpha$ (TNF- $\alpha$ )/TNF receptor 1 (TNFR1), TRAIL/DR4, or TRAIL/DR5] (Figure 2). Ligand and DR interaction triggers recruitment of FADD and initiator pro-caspase- 8 or -10 to form the death-inducing complex (DISC), resulting in activation of these pro-caspases to caspases- 8 and -10 by an autocatalytic process. These caspases in turn activate the effector caspases- $3,-6$, and -7 and cause degradation of the downstream proteins leading to apoptosis. Caspases- 8 and -10 cleave the pro-apoptotic Bcl-2 family member Bid to truncated tBid linking the extrinsic apoptosis pathway to the intrinsic or mitochondrial pathway and trigger cytochrome $c$ release from mitochondria [63-67]. The DR initiated apoptosis pathway is suppressed by the anti-apoptotic protein c-FLIP, which inhibits DISC formation and activation of caspases- 8 and -10 and blocks apoptotic.

In the mitochondrial or intrinsic apoptosis pathway, various apoptotic stimuli (e.g., DNA damaging agents, radiation, conventional chemotherapeutic drugs, and small molecule anticancer compounds) trigger mitochondrial outer membrane permeabilization (MOMP). This process is initiated by activation of $\mathrm{Bcl}-2$ homologous proapoptotic proteins (e.g., Bax, Bak, and Bad) and the Bcl-2 homology domain-3 (BH3)-only family of proteins including Bid, Bim, or PUMA [65-71], and provides an interactive protein network with mitochondria, which leads to the release of apoptosis triggering factors. The apoptosis inducing factors include certain caspases, Smac/DIABLO, and other factors from the mitochondrial intramembrane space to the cytosol. After release from mitochondria, cytochrome $c$ and dATP bind to apoptotic proteinase-activating factor-1 (Apaf-1) to form the apoptosome, and this complex triggers procaspase- 9 autoactivation. The active caspase- 9 in turn activates caspases- $2,-3,-6,-7,-8$, and -10 , leading to degradation of cellular proteins and resulting in apoptosis [6-71].

The third main apoptosis pathway is the endoplasmic reticulum (ER)-mediated apoptosis pathway. The ER promotes the correct folding of proteins and mediates ER-associated degradation of unfolded or misfolded protein. Dysregulation of ER functions triggers an accumulation of unfolded or misfolded proteins in the ER lumen, leading to ER stress (ERS), which induces the unfolded protein response (UPR) or the ERS response (ERSR), resulting in restored homeostasis or apoptosis [75,76].

CSCs display resistance to apoptosis by upregulating the expression of anti-apoptotic proteins including the cellular FLICEinhibitory protein (c-FLIP), the Bcl-2 family of proteins, and inhibitor of apoptosis proteins (IAPs) $[11,77,78]$. CSCs upregulate c-FLIP expression and are resistant to TNF-related apoptosis-inducing ligand (TRAIL)-induced apoptosis [79]. Overexpression of IAPs also plays a crucial role in resistance to TRAIL and chemotherapeutic agents, as well as CSC apoptosis [80].

\section{Mechanisms of CSCs death resistance}

Several mechanisms trigger drug resistance and make CSCs refractory to apoptosis. Characterizing the mechanisms that prevent apoptosis and identifying useful therapeutic targets to increase apoptosis in CSCs are particularly significant for successful cancer therapy. These mechanisms are discussed in detail in the following sections.

\section{Multidrug resistance transporters in CSCs}

Several ABC transporters including P-glycoprotein (P-gp, MDR1, $\mathrm{ABCB} 1$ ), multidrug resistance protein 1 (MRP1, ABCC1), breast cancer resistance protein (BCRP, ABCG2) [81-84], and MRP5/ABCC5 [83], have been extensively investigated as multidrug resistance transporters in tumors. Overexpression of these proteins in several solid tumor types, acute myeloid leukemia (AML), and myeloma lead to ATPdependent efflux of a wide range of conventional chemotherapeutic agents as well as many molecular-targeted cancer drugs, resulting in lower drug levels in the resistant cells below the amount required to trigger cell death [81-84].

Conclusive evidence shows that CSCs in various solid tumors and hematological malignancies upregulate these $\mathrm{ABC}$ transporters, resulting in drug resistance in these cells [85]. Wang et al. [86] reported that Panc-1 pancreatic CSCs displayed resistance to gemcitabine, upregulated expression of CD133/CD44/Oct4/Nestin compared to the parental Panc-1 cells, and overexpressed P-gp and anti-apoptotic proteins. In glioblastoma CSCs, epigallocatechin gallate (EGCG) treatment downregulated P-gp overexpression but not that of ABCG2 or O6- methylguanine-DNA methyltransferase (MGMT) and increased the cytotoxic effect of TMZ [55]. Wilson et al [84] isolated melanoma cancer stem cells (MCSCs) and demonstrated that ABCB5 maintains these cells.

\section{PI3K/Akt/mTOR signaling pathway plays a crucial role in CSCs}

This pathway functions in many important cellular processes and contributes to drug resistance in cancer. Several studies have demonstrated that upregulation of $\mathrm{PI} 3 \mathrm{~K} / \mathrm{Akt} / \mathrm{mTOR}$ plays a pivotal role in the CSC maintenance [87-91]. Emerging data suggest that the $\mathrm{PI} 3 \mathrm{~K} / \mathrm{Akt} / \mathrm{mTOR}$ signaling pathway could be a rational and promising target for the development of anti-CSC drugs including salinomycin, metformin, silibinin E1201, rottlerin, and torin [89]. Moreover, the antidiabetic drug metformin, an inhibitor of PI3K/Akt/mTOR signaling, effectively reduced temozolomide (TMZ) resistance in CSCs [92]. Furthermore, the combination of metformin with sorafenib as a RAF inhibitor also significantly reduced CSC oxidative stress, efflux pump activity, and synergistically killed these cells [93]. CSCs heavily rely on mitochondrial oxidative phosphorylation. Metformin has been shown to use this metabolic weakness and enhance CSC sensitivity to conventional cancer chemotherapies, overcome drug resistance and increase treatment efficacy [94].

\section{Dysregulated anti-apoptotic proteins in CSCs in the Bcl-2 family of proteins}

Apoptosis evasion and the capacity of cancer cells to self-replicate, proliferate, and metastasize are distinct hallmarks of cancer [11,95]. In various cancers, several steps within the extrinsic and intrinsic apoptotic pathways in CSCs may be dysregulated $[11,96]$. However, the abnormal expression levels as well as levels and ratios of apoptotic and antiapoptotic proteins and their contribution to drug resistance in CSCs have not been well characterized and delineated in detail. Bcl-2 family 
proteins consist of the anti-apoptotic molecules Bcl-2, Bcl-XL and Mcl1 and the pro-apoptotic proteins Bax, Bak, Bid, Bim, Bik, Noxa and Puma [97,98]. Increased levels of Bcl-2 family proteins were shown in CSCs and high levels of these proteins are associated with the resistance of CSCs to apoptosis and anticancer drugs $[99,100]$. This resistance is partly due to the ratio of anti- to pro-apoptotic protein levels, which increases cell survival [99]. Moreover, aberrantly increased expression of nuclear factor erythroid 2-related factor 2 (Nrf2), the redux-sensing transcription factor promotes CSC survival by elevating transcription of ABCG2, Bcl-2, and Bmi-1, a member of the Polycomb Repressor Complex (PRC1) genes [101]. Since expression of the Bcl-2 family of anti-apoptotic proteins is significant for cell survival and resistance to apoptosis and drugs in CSCs, therapeutic interventions to eliminate CSCs using inhibitors of these proteins have been actively pursued.

\section{TRADD and NF- $\kappa B$}

The tumor necrosis factor receptor 1 (TNFR1)-associated death domain protein (TRADD) is an adaptor protein in TNFR1 signaling and participates in NF- $\mathrm{BB}$ activation as well as survival signaling in CSCs [65] downstream of DR4, DR5 (Figure 2). Moreover, TRAIL has been shown to promote the formation of the intracellular Complex II composed of FADD, TRADD, caspase-8, caspase-10, RIP1, TRAF2 and IKK-y [102]. Cancer cells and CSCs often have constitutively activated transcription factor NF- $\mathrm{KB}$ that promotes expression levels of apoptosis inhibitory proteins and drug resistantance proteins and provides enhanced survival and resistance to therapies in cancer cells. Upregulated expression of TRADD is sufficient to activate NF- $\kappa B$ in glioblastoma (GBM) cancer stem cells (GSCs) [103]. Furthermore, cytoplasmic TRADD expression has been shown to be significantly associated with worse progression-free survival (PFS) in GBM patients. Knockdown of TRADD by shRNA in GSCs triggered decreased NF- $\kappa B$ activity and reduced the viability of these cells, revealing that TRADD is required for maintenance of GBM stem cell populations [103]. NF- $\kappa B$ signaling plays an important role in the maintenance of CSCs [104]. In advanced ovarian cancers, NF- $\kappa \mathrm{B}$ signaling via the RelB transcription factor supports directly regulating the cancer stem-like associated enzyme aldehyde dehydrogenase (ALDH) [105]. Additionally, the $\mathrm{NF}-\kappa \mathrm{B}$ signaling pathway plays a critical role in the chemoresistance of gastric cancer stem cells [105]. NF- $\kappa B$ activity supports CSC maintenance and reduced sensitivity to NF- $\kappa B$ inhibitors, indicating that high activity of NF- $\kappa \mathrm{B}$ plays a critical role in the survival of CSCs [106].

\section{Role of the IAP family in CSC drug and apoptosis resistance}

The IAP family consists of several proteins including survivin, IAP1, cIAP2, X-linked inhibitors of apoptosis (XIAP), ML-IAP, NAIP, and ILP-2 [70-72]. IAPs inhibit the activity of caspases-3, -7, and -9 and participate in the evasion of cancer cells from apoptosis $[77,80]$. Overexpression of IAP family proteins has been shown in solid tumors and hematological malignancies and causes resistance to apoptosis, chemotherapeutics agents, and radiation therapy as well as causing poor prognoses [77]. IAPs function through interactions of their BIR (baculoviral IAP repeat) protein domains and these interactions are antagonized by Smac/Diablo, an inverse regulator for the inhibitors of IAPs and induction of apoptosis $[77,80]$. Survivin plays a role in $\mathrm{CD} 133+$ cell chemoresistance to 5-fluorouracil (5-FU) in colon CSCs and a survivin inhibitor may be a new targeted agent to effectively treat CD133+ colon cancer [107].

The critical roles of IAPs in maintaining CSCs and the importance of IAP inhibitors being more effective for CD133+ stem-like medulloblastoma (MB) CSCs has been shown [108]. Holt et al. [109] showed that pharmacological downregulation of XIAP in pediatric tumor cells triggers apoptosis and sensitizes cells to cytotoxic agents. Furthermore, Ji et al. [110] have shown that XIAP, a member of the IAP family, has a critical role in maintaining CSCs in nasopharyngeal carcinoma (NPC) stem cells. These authors showed that XIAP regulates the stability of the CSC marker Sox2, which is important for the maintenance and self-renewal of NPC CSCs. The important role of IAPs in CSCs was also demonstrated by Janzen et al. [111] showing that the cIAP inhibitor Birinapant overcomes platinum resistance in a CSC population of ovarian cancer in vivo, indicating that IAPs may play a significant role in cancer drug resistance and recurrence.

\section{c-FLIP overexpression in CSCs}

c-FLIP is a catalytically inactive caspase- $8 /-10$ homolog and a master anti-apoptotic protein that suppresses cytokine- and chemotherapyinduced apoptosis and causes resistance to these agents [112]. c-FLIP is expressed as long (c-FLIPL), short (c-FLIPS), and c-FLIPR splice variants in human cells. c-FLIP binds to FADD and/or caspases- 8 or -10 and TRAIL receptor 5 (DR5) and prevents Death-Inducing Signaling Complex (DISC) formation. Moreover, c-FLIPL and c-FLIPS are also known to have multifunctional roles in various signaling pathways, as well as activating and/or upregulating several cytoprotective and prosurvival signaling proteins including Akt, ERK, and NF- $\mathrm{kB}$. It is known that $\mathrm{Ku} 70$, a protein that repairs DNA breaks, stabilizes c-FLIP, which is regulated by acetylation [113]. Several reports have demonstrated that c-FLIP isoforms maintain the survival and resistance of CSCs to apoptosis and anti-cancer therapies [79,114,115]. CD133, a CSC marker that plays a role in CSC tumorigenesis, metastasis and chemoresistance, can also upregulate the expression of c-FLIP in CD133+ cells, thus inhibiting apoptosis $[116,117]$.

\section{Aldehyde dehydrogenase (ALDH) activity}

Aldehyde dehydrogenase (ALDH) isoforms consist of a superfamily of enzymes that detoxify a variety of endogenous and exogenous aldehydes and high ALDH activity has been frequently used as a selectable marker for CSCs $[118,119]$. Much evidence suggests that ALDH may be used as a marker for CSC self-renewal, proliferation, differentiation, and resistance to drugs [118-120]. It is well documented that the ALDH protein family is a hallmark of CSCs and ALDH1A1 is the most studied ALDH isoform [121,122]. Expression of ALDH1 protein in CSCs is a negative prognostic indicator and predictor of poor clinical outcome in cancer patients, and high ALDH activity has been attributed to chemoresistant CSCs in different tumor types $[118,119,121]$. Overall, substantial data indicate a critical role of ALDH, particularly ALDH1, in CSC biology and therapy resistance [118122]. Therefore, inhibition of ALDH activity may be a rational and potentially useful therapeutic strategy for targeting CSCs with the aim of increasing the efficacy of cancer therapies.

\section{Enhanced DNA damage response and ROS scavenging in CSCs}

Much evidence has shown that CSCs are resistant to DNA damaging therapies by regulation of the cell cycle, increasing DNA repair capacity, and effective scavenging of reactive oxygen species (ROS) [123-126]. DNA-damage response (DDR) is considered a significant source of resistance to DNA-damaging treatments and CSCs, and check point inhibitors to sensitize CSCs to DNA-damaging treatments have been developed [127]. Interestingly, DDR appears as a relevant target to sensitize cancer cells and cancer stem cells to classical 
radio- and chemotherapies as well as to overcome resistance [127]. Bartucci et al. [128] reported that in NSCLC, chemotherapy targeting the DNA damage checkpoint (CHK1) signaling in CSCs was p53 independent and caused cell cycle arrest, more efficient DNA damage repair, and enhanced cell survival compared to the bulk of the tumor cell population. Moreover, targeting $\mathrm{CHK} 1$ and PARP1 may provide an effective anti-CSC strategy [126].

\section{Autophagy as a cytoprotective and drug resistance mechanism in CSCs}

Autophagy is a catabolic pathway which is characterized by autophagosome formation and triggers tumor cell survival and drug resistance [129-131]. Autophagy is critical as a survival mechanism in tumors having defects in the apoptotic signaling pathways, and CSCs show a high level of autophagy, which contributes to their survival and therapy resistance [131-133]. Autophagy also determines cell fate by targeting degradation of key transcription factors, including p53 and FoxO3A, or by enforcing quiescent growth arrest [131]. Apart from promoting resistance to chemotherapy, high levels of autophagy in CSCs maintains their pluripotency, allows them to cope with low nutrients and hypoxia in the tumor microenvironment, regulates CSCs migration and invasion, and helps them escape immunosurveillance [132]. Beclin 1, a Bcl-2 homology 3 (BH3) domain only protein, is an essential initiator of autophagy and a critical determinant of whether cells undergo autophagy or apoptosis [134]. The BH3 domain of Beclin 1 interacts with Bcl-2 family members. Therefore, the role of Bcl-2 in inhibiting apoptosis and autophagic cell death makes the Bcl-2 protein and autophagy manipulation excellent targets and strategies to inhibit drug, anti-apoptotic, and autophagy-related resistance mechanisms.

\section{Conclusion}

It well documented that CSCs achieve the hallmarks of malignancy including self-renewal and replicative immortality; resistance to chemotherapeutic agents, radiotherapy, and cell death; invasiveness and tumor recurrence. CSC-related drug resistance mechanisms might be important for predicting patient response to therapies and guiding treatment selection for various tumors. A challenging task for the development of CSC-specific therapeutics is identifying specific biomarkers of CSCs to analyze their population during the course of tumor treatment. Taken together, the foregoing discussion reveals that CSCs can contribute to tumor resistance to chemotherapeutic agents and cell death, provide a better understanding of the molecular mechanisms underlying CSC unresponsiveness to therapies, and may lead to the identification of specific therapeutics and novel strategies to increase the sensitivity of CSCs to cancer therapeutics.

\section{Acknowledgment}

I would like to thank Dr. Mary D. Kraeszig for her editorial assistance. The work in the author's laboratory on drug resistance and apoptosis signaling pathways was supported by research grants from the National Cancer Institute (CA 080734, CA 90878, and CA 101743), and Department of Defense (DOD) (OC 06095).

\section{Conflicts of interest}

The author has no conflicts of interest to declare.

\section{References}

1. Nimmakayala RK, Batra SK, Ponnusamy MP (2019) Unraveling the journey of cancer stem cells from origin to metastasis. Biochim Biophys Acta Rev Cancer 187: 50-63. [Crossref]
2. Zhu P, Fan Z (2018) Cancer stem cells and tumorigenesis. Biophys Rep 4: 178-188.

3. Nowell PC (1976) The clonal evolution of tumor cell populations. Science 194: 23-28.

4. Safa AR, Saadatzadeh MR, Cohen-Gadol AA, Pollok KE, Bijangi-Vishehsaraei K (2015) Glioblastoma stem cells (GSCs) epigenetic plasticity and interconversion between differentiated non-GSCs and GSCs. Genes Dis 2: 152-163.

5. Aderetti DA, Hira VVV, Molenaar RJ, van Noorden CJF (2018) The hypoxic periarteriolar glioma stem cell niche, an integrated concept of five types of niches in human glioblastoma. Biochim Biophys Acta Rev Cancer 1869: 346-354.

6. Valent P, Bonnet D, De Maria R, Lapidot T, Copland M, et al. (2012) Cancer stem cell definitions and terminology: the devil is in the details. Nat Rev Cancer 12: 767-775.

7. O'Connor ML, Xiang D, Shigdar S, Macdonald J, Li Y, et al. (2014) Cancer stem cells: A contentious hypothesis now moving forward. Cancer Lett 344: 180-187.

8. Holohan C, Van Schaeybroeck S, Longley DB, Johnston PG (2013) Cancer drug resistance: an evolving paradigm. Nat Rev Cancer 13: 714-726.

9. Turdo A, Veschi V, Gaggianesi M, Chinnici A, Bianca P, et al. (2019) Meeting the challenge of targeting cancer stem cells. Front Cell Dev Biol 7: 16.

10. Chatterjee N, Bivona TG (2019) Polytherapy and Targeted Cancer Drug Resistance. Trends Cancer 5: 170-182.

11. Safa AR (2016) Resistance to cell death and its modulation in cancer stem cells. Crit Rev Oncog 21: 203-219.

12. Senthebane DA, Jonker T, Rowe A, Thomford NE, Munro D, et al. (2018) The role of tumor microenvironment in chemoresistance: $3 \mathrm{D}$ extracellular matrices as accomplices. Int J Mol Sci 19 E2861.

13. Adorno-Cruz V, Kibria G, Liu X, Doherty M, Junk DJ, et al. (2015) Cancer stem cells targeting the roots of cancer, seeds of metastasis, and sources of therapy resistance. Cancer Res 75: 924-929.

14. Khan IN, Al-Karim S, Bora RS, Chaudhary AG, Saini KS (2015) Cancer stem cells: a challenging paradigm for designing targeted drug therapies. Drug Discov Today 20: $1205-1216$

15. Sørensen MD, Fosmark S, Hellwege S, Beier D, Kristensen BW, et al. (2015) Chemoresistance and chemotherapy targeting stem-like cells in malignant glioma. $A d v$ Exp Med Biol 853: 111-138.

16. da Silva-Diz V, Lorenzo-Sanz L, Bernat-Peguera A, Lopez-Cerda M, Munoz P (2018) Cancer cell plasticity: Impact on tumor progression and therapy response. Semin Cancer Biol 53: 48-58.

17. Foo J, Michor F. (2014) Evolution of acquired resistance to anti-cancer therapy. $J$ Theor Biol 355: 10-20.

18. Tang KD, Ling MT (2014) Targeting drug-resistant prostate cancer with dual PI3K/ mTOR inhibition. Curr Med Chem 21: 3048-3056.

19. Koff JL, Ramachandiran S, Bernal-Mizrachi L (2015) A time to kill: targeting apoptosis in cancer. Int J Mol Sci 16: 2942-2955.

20. Fulda S (2015) Targeting apoptosis for anticancer therapy. Semin Cancer Biol 31: 84 88.

21. Zang F, Wei X, Leng X, Yu M, Sun B (2014) c-FLIP(L) contributes to TRAIL resistance in HER2-positive breast cancer. Biochem Biophys Res Commun 450: 267-273.

22. Safa AR, Pollok KE (2011) Targeting the anti-apoptotic protein c-FLIP for cancer therapy. Cancers (Basel) 3: 1639-1671.

23. Sonbol MB, Ahn DH, Bekaii-Saab T (2019) Therapeutic targeting strategies of cancer stem cells in gastrointestinal malignancies. Biomedicines 7: E17.

24. Saygin C, Matei D, Majeti R, Reizes O, Lathia JD (2019) Targeting cancer stemness in the clinic: from hype to hope. Cell Stem Cell 24: 25-40.

25. Pan Y, Ma S, Cao K, Zhou S, Zhao A, et al. (2018) Therapeutic approaches targeting cancer stem cells. J Cancer Res Ther 14: 1469-1475.

26. Hong M, Tan HY, Li S, Cheung F, Wang N, et al. (2016) Cancer stem cells: the potential targets of Chinese medicines and their active compounds. Int J Mol Sci 17: E893.

27. Steinbichler TB, Dud as J, Skvortsov S, Ganswindt U, Riechelmann H, Skvortsova II (2018) Therapy resistance mediated by cancer stem cells. Semin Cancer Biol 53: 156-167.

28. Begicevic RR, Falasca M (2017) ABC transporters in cancer stem cells: beyond chemoresistance. Int J Mol Sci 18: E2362. 
29. Batlle E, Clevers H (2017) Cancer stem cells revisited. Nat Med 23: 1124-1134.

30. Takeishi S, Nakayama KI (2016) To wake up cancer stem cells, or to let them sleep, that is the question. Cancer Science 107:875-881.

31. Vassalli G (2019) Aldehyde dehydrogenases: Not just markers, but functional regulators of stem cells. Stem Cells Int 2019: 3904645.

32. Najafi M, Farhood B, Mortezaee K (2019) Cancer stem cells (CSCs) in cancer progression and therapy. J Cell Physiol 234: 8381-8395.

33. Wang R, Sun Q, Wang P, Liu M, Xiong S, et al. (2016) Notch and Wnt/ $\beta$-catenin signaling pathway play important roles in activating liver cancer stem cells. Oncotarget 7: $5754-5768$.

34. Safa AR, Saadatzadeh MR, Cohen-Gadol AA, Pollok KE, Bijangi-Vishehsaraei K (2016) Emerging targets for glioblastoma stem cell therapy. J Biomed Res 30: 19-31.

35. Yuan ZX, Mo J, Zhao G, Shu G, Fu HL, et al. (2016) Targeting strategies for renal cell carcinoma: from renal cancer cells to renal cancer stem cells. Front Pharmacol 7: 423.

36. Luo M, Wicha MS (2019) Targeting cancer stem cell redox metabolism to enhance therapy responses. Semin Radiat Oncol 29: 42-54.

37. Snyder V, Reed-Newman TC, Arnold L, Thomas SM, Anant S (2018) Cancer stem cell metabolism and potential therapeutic targets. Front Oncol 8: 203.

38. Somasagara RR, Spencer SM, Tripathi K, Clark DW, Mani C, et al. (2017) RAD6 promotes DNA repair and stem cell signaling in ovarian cancer and is a promising therapeutic target to prevent and treat acquired chemoresistance. Oncogene 36: 66806690 .

39. Macha MA, Rachagani S, Qazi AK, Jahan R, Gupta S, et al. (2017) Afatinib radiosensitizes head and neck squamous cell carcinoma cells by targeting cancer stem cells. Oncotarget 8: 20961-20973.

40. Colak S, Medema JP (2014) Cancer stem cells_-important players in tumor therapy resistance. FEBS J 281: 4779-4791.

41. Prieto-Vila M, Takahashi RU, Usuba W, Kohama I, Ochiya T (2017) Drug resistance driven by cancer stem cells and their niche. Int J Mol Sci 18: E2574.

42. Yeldag G, Rice A2, Del Río Hern andez A (2018) Chemoresistance and the selfmaintaining tumor microenvironment. Cancers (Basel) 10: E471.

43. Dey P, Rathod M, De A (2019) Targeting stem cells in the realm of drug-resistant breast cancer. Breast Cancer (Dove Med Press) 11: 115-135.

44. Suresh R, Ali S, Ahmad A, Philip PA, Sarkar FH (2016) The role of cancer stem cells in recurrent and drug-resistant lung cancer. Adv Exp Med Biol 890: 57-74.

45. Toledo-Guzm an ME, Bigoni-Ordóñez GD, Ib añez Hernandez M, Ortiz-Sanchez E (2018) Cancer stem cell impact on clinical oncology. World J Stem Cells 10: 183-195.

46. Sharifzad F, Ghavami S, Verdi J, Mardpour S, Mollapour Sisakht M, et al. (2019) Glioblastoma cancer stem cell biology: Potential theranostic targets. Drug Resist Updat 42: $35-45$.

47. Wong ALA, Bellot GL, Hirpara JL, Pervaiz S (2019) Understanding the cancer stem cell phenotype: A step forward in the therapeutic management of cancer. Biochem Pharmacol 162: 79-88.

48. Ajani JA, Song S, Hochster HS, Steinberg IB (2025) Cancer stem cells: the promise and the potential. Semin Oncol 42 Suppl 1: S3-17.

49. Jhanwar-Uniyal M, Amin AG, Cooper JB, Das K, Schmidt MH, et al. (2017) Discrete signaling mechanisms of mTORC1 and mTORC2: connected yet apart in cellular and molecular aspects. Adv Biol Regul 64: 39-48.

50. Wang YH, Scadden DT (2015) Harnessing the apoptotic programs in cancer stem-like cells. EMBO Rep 16: 1084-1098.

51. Saeg F, Anbalagan M (2018) Breast cancer stem cells and the challenges of eradication: a review of novel therapies. Stem Cell Investig 5: 39.

52. Wen Y, Cai J, Hou Y, Huang Z, Wang Z (2017) Role of EZH2 in cancer stem cells: from biological insight to a therapeutic target. Oncotarget 8: 37974-37990.

53. Cai MH, Xu XG, Yan SL, Sun Z, Ying Y, et al. (2018) Depletion of HDAC1, 7 and 8 by histone deacetylase inhibition confers elimination of pancreatic cancer stem cells in combination with gemcitabine. Sci Rep 8: 1621.

54. Yang W, Liu Y, Gao R, Yu H, Sun T (2018) HDAC6 inhibition induces glioma stem cells differentiation and enhances cellular radiation sensitivity through the SHH/Gli1 signaling pathway. Cancer Lett 415: 164-176.
55. Iriondo O, R abano M, Domenici G, Carlevaris O, Lopez-Ruiz JA, et al. (2015) Distinct breast cancer stem/progenitor cell populations require either HIF1 $\alpha$ or loss of PHD3 to expand under hypoxic conditions. Oncotarget 6: 31721-31739.

56. Lee G, Auffinger B, Guo D, Hasan T, Deheeger M, et al. (2016) Dedifferentiation of glioma cells to glioma stem-like cells by therapeutic stress-induced HIF signaling in the recurrent GBM model. Mol Cancer Ther 15: 3064-3076.

57. Axelson H, Fredlund E, Ovenberger M, Landberg G, Påhlman S (2005) Hypoxiainduced dedifferentiation of tumor cells - a mechanism behind heterogeneity and aggressiveness of solid tumors. Semin Cell Dev Biol 16: 554-563.

58. Del Vecchio CA, Feng Y, Sokol ES, Tillman EJ, Sanduja S, et al. (2014) Dedifferentiation confers multidrug resistance via noncanonical PERK-Nrf2 signaling. PLoS Biol 12: e1001945.

59. Ganguly R, Mohyeldin A, Thiel J, Kornblum HI, Beullens M, et al. (2015) MELK-a conserved kinase: functions, signaling, cancer, and controversy. Clin Transl Med 4: 11.

60. Kim SH, Joshi K, Ezhilarasan R, Myers TR, Siu J, et al., (2015) EZH2 protects glioma stem cells from radiation-induced cell death in a MELK/FOXM1-dependent manner. Stem Cell Reports 4: 226-238.

61. Inoue H, Kato T, Olugbile S, Tamura K, Chung S, et al. (2016) Effective growthsuppressive activity of maternal embryonic leucine-zipper kinase (MELK) inhibitor against small cell lung cancer. Oncotarget 7: 13621-13633.

62. Ingangi V, Minopoli M, Ragone C, Motti ML, Carriero MV (2019) Role of microenvironment on the fate of disseminating cancer stem cells. Front Oncol 9: 82 .

63. Perusina Lanfranca M, Thompson JK, Bednar F, Halbrook C, Lyssiotis C, et al. (2018) Metabolism and epigenetics of pancreatic cancer stem cells. Semin Cancer Biol S1044579X: 30116-30120.

64. Pires BR, DE Amorim IS, Souza LD, Rodrigues JA, Mencalha AL (2016) Targeting cellular signaling pathways in breast cancer stem cells and its implication for cancer treatment. Anticancer Res 36: 5681-5691.

65. Grimm S (2012) The ER-mitochondria interface: the social network of cell death Biochim Biophys Acta 1823: 327-34.

66. Shakeri R, Kheirollahi A, Davoodi J (2017) Apaf-1: Regulation and function in cell death. Biochimie 135: 111-125.

67. Edlich F (2018) BCL-2 proteins and apoptosis: recent insights and unknowns. Biochem Biophys Res Commun 500: 26-34.

68. Aouacheria A, Baghdiguian S, Lamb HM, Huska JD, Pineda FJ, et al. (2017) Connecting mitochondrial dynamics and life-or-death events via Bcl-2 family proteins. Neurochem Int 109: 141-161.

69. Vela L, Marzo I (2015) Bcl-2 family of proteins as drug targets for cancer chemotherapy: the long way of BH3 mimetics from bench to bedside. Curr Opin Pharmacol 23: 74-81.

70. Correia C, Lee SH, Meng XW, Vincelette ND, Knorr KL, et al. (2015) Emerging understanding of Bcl-2 biology: implications for neoplastic progression and treatment. Biochim Biophys Acta 1853: 1658-1671.

71. Krammer PH, Kamiński M, Kiessling M, Gülow K (2007) No life without death. Adv Cancer Res 97: 111-138.

72. Lavrik IN, Krammer PH (2012) Regulation of CD95/Fas signaling at the DISC. Cell Death Differ 19: 36-41.

73. Lavrik I, Golks A, Krammer PH (2005) Death receptor signaling. J Cell Sci 118: 265 267.

74. Redza-Dutordoir M, Averill-Bates DA (2016) Activation of apoptosis signalling pathways by reactive oxygen species. Biochim Biophys Acta 1863: 2977-2992.

75. Zhang Z, Zhang L, Zhou L, Lei Y, Zhang Y, et al. (2018) Redox signaling and unfolded protein response coordinate cell fate decisions under ER stress. Redox Biol S22132317: 30895-30894.

76. Bahar E, Kim JY, Yoon H (2019) Chemotherapy resistance explained through endoplasmic reticulum stress-dependent signaling. Cancers (Basel) 11: E338.

77. Mohamed MS, Bishr MK, Almutairi FM, Ali AG (2017) Inhibitors of apoptosis: clinical implications in cancer. Apoptosis 22: 1487-1509.

78. Mukherjee N, Schwan JV, Fujita M, Norris DA, Shellman YG. (2015) Alternative treatments for melanoma: targeting BCL-2 family members to de-bulk and kill cancer stem cells. J Invest Dermatol 135: 2155-2161.

79. French R, Hayward O, Jones S, Yang W, Clarkson R (2015) Cytoplasmic levels of cFLIP determine a broad susceptibility of breast cancer stem/progenitor-like cells to TRAIL. Mol Cancer 14: 209. 
80. Fulda S (2013) Regulation of apoptosis pathways in cancer stem cells. Cancer Lett 338: 168-173.

81. Robey RW, Pluchino KM, Hall MD, Fojo AT, Bates SE, et al. (2018) Revisiting the role of ABC transporters in multidrug-resistant cancer. Nat Rev Cancer 18: 452-464.

82. Lu JF, Pokharel D, Bebawy M (2015) MRP1 and its role in anticancer drug resistance. Drug Metab Rev 47: 406-419.

83. Jaramillo AC1, Cloos J2, Lemos C3, Stam RW, Kaspers GJL, et al. (2019) Ex vivo resistance in childhood acute lymphoblastic leukemia: correlations between BCRP, MRP1, MRP4 and MRP5 ABC transporter expression and intracellular methotrexate polyglutamate accumulation. Leuk Res 79: 45-51

84. Wilson BJ, Saab KR, Ma J, Schatton T, Pütz P, et al. (2014) ABCB5 maintains melanomainitiating cells through a proinflammatory cytokine signaling circuit. Cancer Res 74: 4196-41207.

85. Speigl L, Janssen N, Weide B, Pawelec G, Shipp C (2017) Prognostic impact of the putative cancer stem cell markers ABCG2, CD133, ALDH1A1 and CD44V7/8 in metastatic melanoma. Br J Dermatol 177: 1447-1449.

86. Wang D, Zhu H, Zhu Y, Liu Y, Shen H, et al. CD133(+)/CD44(+)/Oct4(+)/Nestin(+) stemlike cells isolated from Panc-1 cell line may contribute to multi-resistance and metastasis of pancreatic cancer. Acta Histochem 115: 349-356.

87. Wang S, Tang L, Lin J, Shen Z, Yao Y, et al. (2018) ABCB5 promotes melanoma metastasis through enhancing NF- $\mathrm{kB}$ p65 protein stability. Biochem Biophys Res Commun 492: 18-26.

88. Guo Q, Grimmig T, Gonzalez G, Giobbie-Hurder A, Berg G, et al. (2018) ATP-binding cassette member B5 (ABCB5) promotes tumor cell invasiveness in human colorectal cancer. J Biol Chem 293: 11166-11178.

89. Xia P, Xu XY (2015) PI3K/Akt/mTOR signaling pathway in cancer stem cells: from basic research to clinical application. Am J Cancer Res 5: 1602-1609.

90. Yang Q, Jiang W, Hou P (2019) Emerging role of PI3K/AKT in tumor-related epigenetic regulation. Semin Cancer Biol S1044-579X: 30136-30136.

91. Noorolyai S, Shajari N, Baghbani E, Sadreddini S, Baradaran B (2019) The relation between PI3K/AKT signalling pathway and cancer. Gene 698: 120-128.

92. Yang SH, Li S, Lu G, Xue H, Kim DH, et al. (2016) Metformin treatment reduces temozolomide resistance of glioblastoma cells. Oncotarget 7: 78787-78803.

93. Aldea MD, Petrushev B, Soritau O, Tomuleasa CI, Berindan-Neagoe I, et al. (2014) Metformin plus sorafenib highly impacts temozolomide resistant glioblastoma stemlike cells. J BUON 19: 502-511.

94. Mayer MJ, Klotz LH, Venkateswaran V (2015) Metformin and prostate cancer stem cells: a novel therapeutic target. Prostate Cancer Prostatic Dis 18: 303-309.

95. Rosa R, D'Amato V, De Placido S, Bianco R. (2016) Approaches for targeting cancer stem cells drug resistance. Expert Opin Drug Discov 11: 1201-1212.

96. Qiu H, Fang X, Luo Q, Ouyang G (2015) Cancer stem cells: a potential target for cancer therapy. Cell Mol Life Sci 72: 3411-3424.

97. Hata AN, Engelman JA, Faber AC (2015) The BCL2 family: key mediators of the apoptotic response to targeted anticancer therapeutics. Cancer Discov 5: 475-487.

98. Knight T, Luedtke D, Edwards H, Taub JW4, Ge Y (2019) A delicate balance - The BCL-2 family and its role in apoptosis, oncogenesis, and cancer therapeutics. Biochem Pharmacol 162: 250-261.

99. Hu Y, Yagüe E, Zhao J, Wang L, Bai J, et al. (2018) Sabutoclax, pan-active BCL-2 protein family antagonist, overcomes drug resistance and eliminates cancer stem cells in breast cancer. Cancer Lett 423: 47-59.

100. Zhang L, Zhou Y, Chen K, Shi P, Li Y, et al. (2017) The pan-Bc12 inhibitor AT101 activates the intrinsic apoptotic pathway and causes DNA damage in acute myeloid leukemia stem- like cells. Target Oncol 12: 677-12687.

101. Singh R, Letai A, Sarosiek K (2019) Regulation of apoptosis in health and disease: the balancing act of BCL-2 family proteins. Nat Rev Mol Cell Biol 20: 175-193.

102. Wajant H, Scheurich P (2011) TNFR1-induced activation of the classical NF- $\kappa B$ pathway. FEBS J 278: 862-876.

103. Chakraborty S, Li L, Tang H, Xie Y, Puliyappadamba VT, et al. (2013) Cytoplasmic TRADD confers a worse prognosis in glioblastoma. Neoplasia 15: 888-897.

104. Rinkenbaugh AL, Baldwin AS (2016) The NF- $\kappa B$ pathway and cancer stem cells. Cells 5: E16.
105. Fu ZH, Liu SQ, Qin MB, Huang JA, Xu CY, et al. (2018) NIK and IKK $\beta$ binding protein contributesto gastric cancer chemoresistance by promoting epithelial

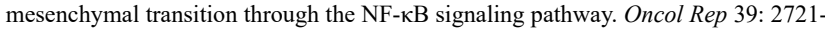
2730 .

106. Zhou J, Zhang H, Gu P, Bai J, Margolick JB, et al. (2018) NF-אB pathway inhibitors preferentially inhibit breast cancer stem-like cells. Breast Cancer Res Treat 111: 419427.

107. Lee MR, Ji SY, Mia-Jan K, Cho MY (2015) Chemoresistance of CD133(+) colon cancer may be related with increased survivin expression. Biochem Biophys Res Commun 463: 229-234.

108. Chen SM, Li YY, Tu CH, Salazar N, Tseng YY, et al. (2016) Blockade of inhibitor of apoptosis proteins in combination with conventional chemotherapy leads to synergistic antitumor activity in medulloblastoma and cancer stem-like cells. PLoS One 11: e 0161299 .

109. Holt SV, Brookes KE, Dive C, Makin GW (2011) Down-regulation of XIAP by AEG35156 in paediatric tumour cells induces apoptosis and sensitises cells to cytotoxic agents. Oncol Rep 25: 1177-1181.

110. Ji J, Yu Y, Li ZL, Chen MY, Deng R, et al. (2018) XIAP limits autophagic degradation of Sox 2 and is a therapeutic target in nasopharyngeal carcinoma stem cells Theranostics 8: 1494-1510.

111. Janzen DM, Tiourin E, Salehi JA, Paik DY, Lu J, et al. (2016) An apoptosis-enhancing drug overcomes platinum resistance in a tumour-initiating subpopulation of ovarian cancer. Nat Commun 7: 10703

112. Safa AR (2013) Roles of c-FLIP in apoptosis, necroptosis, and autophagy. J Carcinog Mutagen 6: 003.

113. Gong P, Wang Y, Jing Y (2019) Apoptosis induction by histone deacetylase inhibitors in cancer cells: role of Ku70. Int J Mol Sci 20: E1601.

114. Yoon MJ, Kang YJ, Kim IY, Kim EH, Lee JA, et al. (2013) Monensin, a polyether ionophore antibiotic, overcomes TRAIL resistance in glioma cells via endoplasmic reticulum stress, DR5 upregulation and c-FLIP downregulation. Carcinogenesis 34: 1918-1928.

115. Ding L, Yuan C, Wei F, Wang G, Zhang J, et al. (2011) Cisplatin restores TRAIL apoptotic pathway in glioblastoma-derived stem cells through up-regulation of DR5 and downregulation of c-FLIP. Cancer Invest 29: 511-520.

116. Zobalova R, Stantic M, Prokopova K, Dong LF, Neuzil J (2008) Cancer cells with high expression of CD133 exert FLIP upregulation and resistance to TRAIL-induced apoptosis. Biofactors 34: 231-235.

117. Barzegar Behrooz A, Syahir A, Ahmad S. (2019) CD133: beyond a cancer stem cell biomarker. J Drug Target 27: 257-269.

118. Muzio G, Maggiora M, Paiuzzi E, Oraldi M, Canuto RA (2012) Aldehyde dehydrogenases and cell proliferation. Free Radic Biol Med 52: 735-746.

119. Marcato P, Dean CA, Giacomantonio CA, Lee PW (2011) Aldehyde dehydrogenase: its role as a cancer stem cell marker comes down to the specific isoform. Cell Cycle 10: $1378-1384$.

120. Tomita H, Tanaka K, Tanaka T, Hara A (2016) Aldehyde dehydrogenase $1 \mathrm{~A} 1$ in stem cells and cancer. Oncotarget 7: 11018-11032.

121. Roy M, Connor J, Al-Niaimi A, Rose SL, Mahajan A (2018) Aldehyde dehydrogenase 1A1 (ALDH1A1) expression by immunohistochemistry is associated with chemorefractoriness in patients with high-grade ovarian serous carcinoma. Hum Pathol 73 : $1-6$.

122. Moreb JS. (2008) Aldehyde dehydrogenase as a marker for stem cells. Curr Stem Cell Res Ther 3: 237-246.

123. Manic G, Sistigu A, Corradi F, Musella M, De Maria R, et al. (2018) Replication stress response in cancer stem cells as a target for chemotherapy. Semin Cancer Biol 53: 31- 41 .

124. Ding S, Li C, Cheng N, Cui X, Xu X, et al. (2015) Redox regulation in cancer stem cells. Oxid Med Cell Longev 2015: 750798.

125. Maugeri-Saccà M, Bartucci M, De Maria R. DNA damage repair pathways in cancer stem cells. Mol Cancer Ther 11: 1627-1636.

126. Coudre C, Alani J, Ritchie W, Marsaud V, Sola B, et al. (2016) HIF-1 $\alpha$ and rapamycin act as gerosuppressant in multiple myeloma cells upon genotoxic stress. Cell Cycle 15: $2174-2182$.

127. Ronco C, Martin AR, Demange L, Benhida R (2016) ATM, ATR, CHK1, CHK2 and WEE1 inhibitors in cancer and cancer stem cells. Medchemcomm 8: 295-319. 
128. Bartucci M, Svensson S, Romania P, Dattilo R, Patrizii M, et al. (2012) Therapeutic targeting of Chk1 in NSCLC stem cells during chemotherapy. Cell Death Differ 19: $768-778$.

129. Levine B, Kroemer G (2008). Autophagy in the pathogenesis of disease. Cell 132: 27.

130. White E, DiPaola RS (2009). The double-edged sword of autophagy modulation in cancer. Clin Cancer Res 15: 5308-5316.

131. Smith AG, Macleod KF (2019). Autophagy, cancer stem cells and drug resistance. $J$ Pathol 247: 708-718
132. Rothe K, Porter V, Jiang X (2019) Current outlook on autophagy in human leukemia: foe in cancer stem cells and drug resistance, friend in new therapeutic interventions. Int J Mol Sci 20: E461.

133. Nazio F, Bordi M, Cianfanelli V, Locatelli F, Cecconi F, et al. (2019). Autophagy and cancer stem cells: molecular mechanisms and therapeutic applications. Cell Death Differ 26: 690-702.

134. Marquez RT, Xu L (2012) Bcl-2: Beclin 1 complex: multiple, mechanisms regulating autophagy/apoptosis toggle switch. Am J Cancer Res 2: 214-221.

Copyright: @2019 Safa AR. This is an open-access article distributed under the terms of the Creative Commons Attribution License, which permits unrestricted use, distribution, and reproduction in any medium, provided the original author and source are credited. 